\title{
EPIDEMIOLOGIA DO SURTO DE DOENÇA POR CORONAVÍRUS (COVID-19)
}

The epidemiology of coronavirus disease (COVID-19) outbreak

Epidemiología del brote de la enfermedad por coronavirus (COVID-19)

\section{Raimundo Gonçalves Ferreira Netto ${ }^{1}$, José Wilson do Nascimento Corrêa ${ }^{* 2}$

${ }^{1}$ Departamento de Ensino, Instituição Federal do Amazonas, Manaus, Brasil.

${ }^{2}$ Laboratório de Farmacologia Experimental, Departamento de Ciências Fisiológicas, Instituto de Ciências

Biológicas, Universidade Federal do Amazonas, Manaus, Brasil.

*Correspondência: Laboratório de Farmacologia, Departamento de Ciências Fisiológicas, ICB2, 2 o andar, Setor sul, Campus da Universidade Federal do Amazonas (UFAM), Manaus, Brasil., Av. Rodrigo Otávio, 6200, Coroado I, Manaus, Amazonas, Brasil.CEP:69.080-900.e-mail jwcorrea@ufam.edu.br

Artigo recebido em 07/04/2020 aprovado em 21/04/2020 publicado em 22/04/2020.

\section{RESUMO}

Desde os relatos vindos da China de pneumonia de origem não conhecida, passando pela identificação do novo coronavírus SARS-Cov-2 como agente causador da doença COVID-19, o mundo enfrenta grandes desafios para conter seu avanço e elucidar seu tratamento. O vírus é capaz de ser transmitido de pessoa a pessoa por gotículas originárias de nariz e boca de pacientes infectados ou por contato com superfícies contaminadas. Os sintomas da infecção podem evoluir de tosse, febre e congestão nasal à pneumonia grave. Parcela de indivíduos infectados pode permanecer assintomática e contribuir com propagação do vírus, especialmente para idosos e indivíduos com outras comorbidades que são mais susceptíveis às manifestações graves da doença. O número de novos casos confirmados está em uma crescente, o que reforça a necessidade de manutenção de medidas sanitárias. As projeções para novos casos e, em especial, para o número de mortes causadas por formas graves de COVID-19 para o Brasil são preocupantes e variam de 44.000 a aproximadamente 1.100 .000 mil mortes, a depender das medidas tomadas. Este artigo revisa este cenário e relata as estratégias recomendadas pela OMS aos países. Particularmente, discute a cronologia e o cenário atual da COVID-19 no Brasil.

Palavras-chave: Epidemiologia, COVID-19, SARS-Cov-2.

\section{ABSTRACT}

Since the reports of pneumonia of unknown origin coming from China, to the identification of the new coronavirus SARS-Cov-2 as the causative agent of the disease COVID-19, the world faces great challenges to contain the progress of the disease and elucidate its treatment. The virus is capable of being transmitted from person to person by droplets from the nose and mouth of infected patients or by contact with contaminated surfaces. Symptoms of the infection may progress from cough, fever and nasal congestion to severe pneumonia. A portion of infected individuals may remain asymptomatic and contribute to the spread of the virus, especially for the elderly and individuals with other comorbidities that are more susceptible to severe manifestations of the disease. The number of new confirmed cases is increasing, which reinforces the need to maintain sanitary measures. The projections for new cases and, in particular, for the number of deaths caused by severe forms of COVID-19 for Brazil are worrying and vary from 44,000 to approximately 1,100,000 deaths, depending on the measures taken. This article reviews this scenario and reports on the strategies recommended by WHO to countries. In particular, it discusses the chronology and the current scenario of COVID-19 in Brazil.

Keywords: Epidemiology, COVID-19, SARS-Cov-2. 


\section{RESUMEN}

Desde los informes de China de neumonía de origen desconocido, hasta la identificación del nuevo coronavirus SARS-Cov-2 como agente causante de la enfermedad COVID-19, el mundo enfrenta grandes desafios para contener su progreso y dilucidar su tratamiento. El virus puede transmitirse de persona a persona mediante gotas de la nariz y la boca de pacientes infectados o por contacto con superficies contaminadas. Los sintomas de la infección pueden progresar desde tos, fiebre y congestión nasal hasta neumonía grave. Una parte de las personas infectadas puede permanecer asintomática y contribuir a la propagación del virus, especialmente para los ancianos y las personas con otras comorbilidades que son más susceptibles a las manifestaciones graves de la enfermedad. El número de nuevos casos confirmados está aumentando, lo que refuerza la necesidad de mantener medidas sanitarias. Las proyecciones para nuevos casos y, en particular, para el número de muertes causadas por formas graves de COVID19 para Brasil son preocupantes y varían de 44,000 a aproximadamente 1,100,000 muertes, dependiendo de las medidas tomadas. Este artículo revisa este escenario e informa las estrategias recomendadas por la OMS a los países. En particular, analiza la cronología y el escenario actual de COVID-19 en Brasil.

Descriptores: Epidemiología, COVID-19, SARS-Cov-2.

\section{INTRODUÇÃO}

Primeiros relatos de pneumonia de etiologia não conhecida foram identificados em Wuhan, cidade da Província Chinesa de Hubei em dezembro de 2019 (WU et al., 2020). Em 29 de março de 2020 mais de 700000 casos já foram confirmados em todo o mundo. Estes relatos indicam a rapidez de espalhamento da nova doença por coronavírus (COVID-19) causada pela infecção pelo vírus SAR-COV-2, categorizada como uma pandemia em 11 de março de 2020 pela Organização Mundial de Saúde (OMS). A disseminação do vírus no Brasil e no mundo são objetos deste artigo.

\section{O VÍRUS E AS MANIFESTAÇÕES RELEVANTES DA INFECÇÃO}

Designado anteriormente como 2019-nCOV pela OMS, o SARS-CoV-2 assim identificado pelo "International Committee on Taxonomy of Viruses" é um novo Betacoronavírus que infecta os seres humanos. Baseado em sua similaridade genética a dois outros coronavírus semelhantes ao SARS (vírus causador de Síndrome Respiratória Aguda Severa), sua origem tem sido atribuída a morcegos (ZHOU et al., 2020).

O SARS-CoV-2 aparentemente conseguiu fazer a transição de animais para humanos no mercado DOI: http://dx.doi.org/10.20873/uftsuple2020-8710 de frutos do mar de Huanan em Wuhan, China. No entanto, a rota exata da transmissão precisa urgentemente ser esclarecida (VELAVAN e MEYER, 2020).

A transmissão do vírus de pessoa para pessoa se dá por gotículas, quando o vírus é carreado em pequenas gotículas originárias do nariz e boca de pessoas infectadas ao falar, exalar, tossir ou espirrar. A infecção também pode ocorrer quando uma pessoa toca superfícies ou objetos contaminados e, dessa forma, toca seus olhos, nariz ou boca. Estima-se que o período de incubação de SAR-COV-2 é de 14 dias, com mediana de 4 a 6 dias, embora há relatos de períodos de incubação de até 24 dias (BAI et al., 2020). Períodos de incubação longos podem impactar negativamente sobre os resultados esperados para as políticas de quarentena que objetivam conter a disseminação do vírus.

A proporção de indivíduos infectados por SARS-CoV2 que permanecem assintomáticos ao longo do curso da infecção ainda não está plenamente esclarecida. Em pacientes sintomáticos, as manifestações clínicas da doença geralmente começam após menos de uma semana, consistindo em febre, tosse, congestão nasal, fadiga e outros sinais de infecções do trato respiratório superior. Presença de sintomas gastrointestinais como náusea ou vômito também foram relatadas. Infecções assintomáticas foram relatadas em crianças (CHAN et

Revista Desafios - v7, n. Supl. COVID-19, 2020 
al., 2020). Presença de comorbidades como hipertensão arterial e doença pulmonar obstrutiva crônica também foram relatados em parcela significativa dos pacientes infectados. A infecção pode progredir para doença grave com dispneia e sintomas torácicos graves correspondentes a pneumonia, especialmente em pacientes mais idosos. Achados radiográficos anormais foram observados por tomografia computadorizada na admissão hospitalar (GUAN et al., 2020). A pneumonia ocorre principalmente na segunda ou terceira semana de uma infecção sintomática. Os sinais proeminentes de pneumonia viral incluem diminuição da saturação de oxigênio, desvios de gases no sangue, alterações visíveis através de raios $\mathrm{X}$ do tórax e outras técnicas de imagem. A linfopenia parece ser comum e os marcadores inflamatórios (proteína $\mathrm{C}$ reativa $\mathrm{e}$ citocinas pró-inflamatórias) são elevados. O termo COVID-19 foi aplicado a pacientes que têm casos sintomáticos confirmados em laboratório, sem manifestações radiológicas aparentes. É necessário um melhor entendimento do espectro da doença, uma vez que em uma parcela dos pacientes a infecção por SARS-CoV-2 foi detectada antes do desenvolvimento de pneumonia viral ou a pneumonia viral não se desenvolveu.

\section{CENÁRIO EPIDEMIOLÓGICO MUNDIAL}

A cronologia mundial das infecções por COVID-19 vai além dos objetivos deste texto, mas foi objetivamente relatada por Rothan e colaboradores (ROTHAN e BYRAREDDY, 2020). O total de casos muda diariamente e pode ser observado quase que em tempo real em diversas fontes, entre elas os boletins da OMS (WHO, 2020a) e em website mantido pela Universidade Johns Hopkins, que até 29 de março de 2020 reportou mais de 713000 casos de COVID-19 confirmados e mais de 33500 mortes ao redor do mundo (JHUM, 2020) (Fig.1).

Figura 1. Total de casos confirmados de COVID-19 no mundo (Fonte: JHUM, 2020)

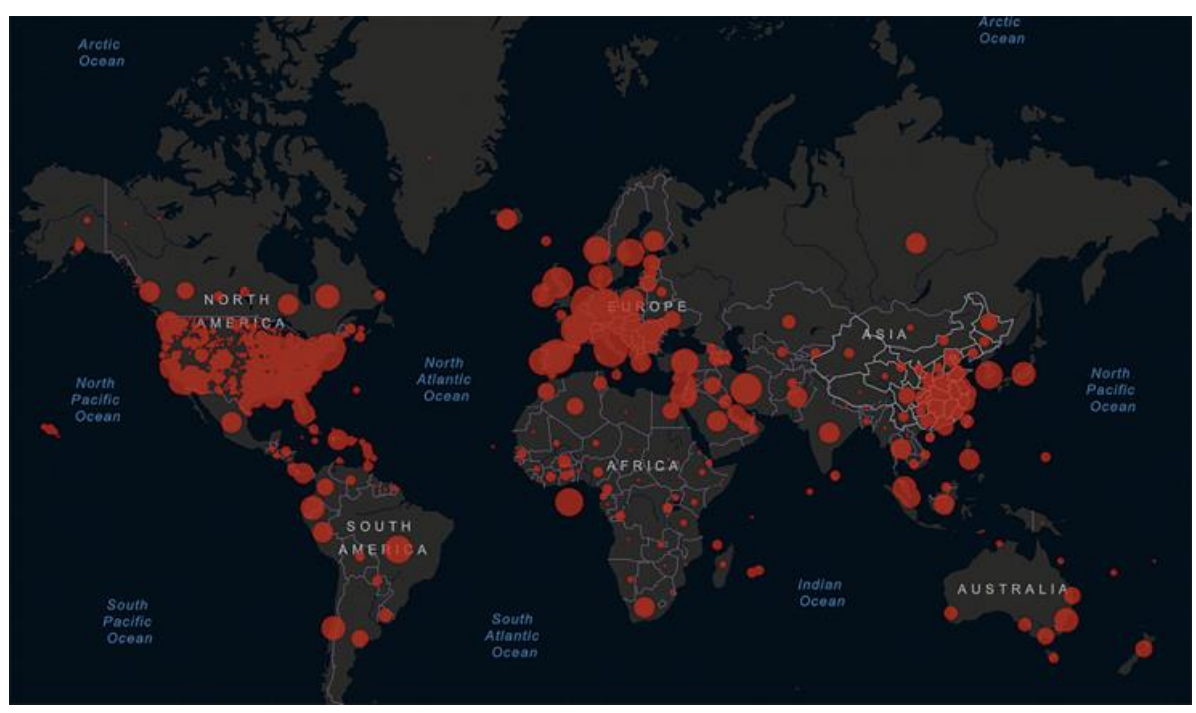

Até 29 de março de 2020, os Estados Unidos, Itália e Espanha figuravam entre os países com o maior número de novos casos identificados nos últimos 7 dias, seguidos por Alemanha, Irã, França e Reino Unido. Nesta lista, o Brasil ocupava o $19^{\circ}$ lugar (Fig.2). 
Figura 2. Total de novos casos confirmados de COVID-19 entre 22 e 29 de março de 2020 (Fonte:

WHO, 2020b)

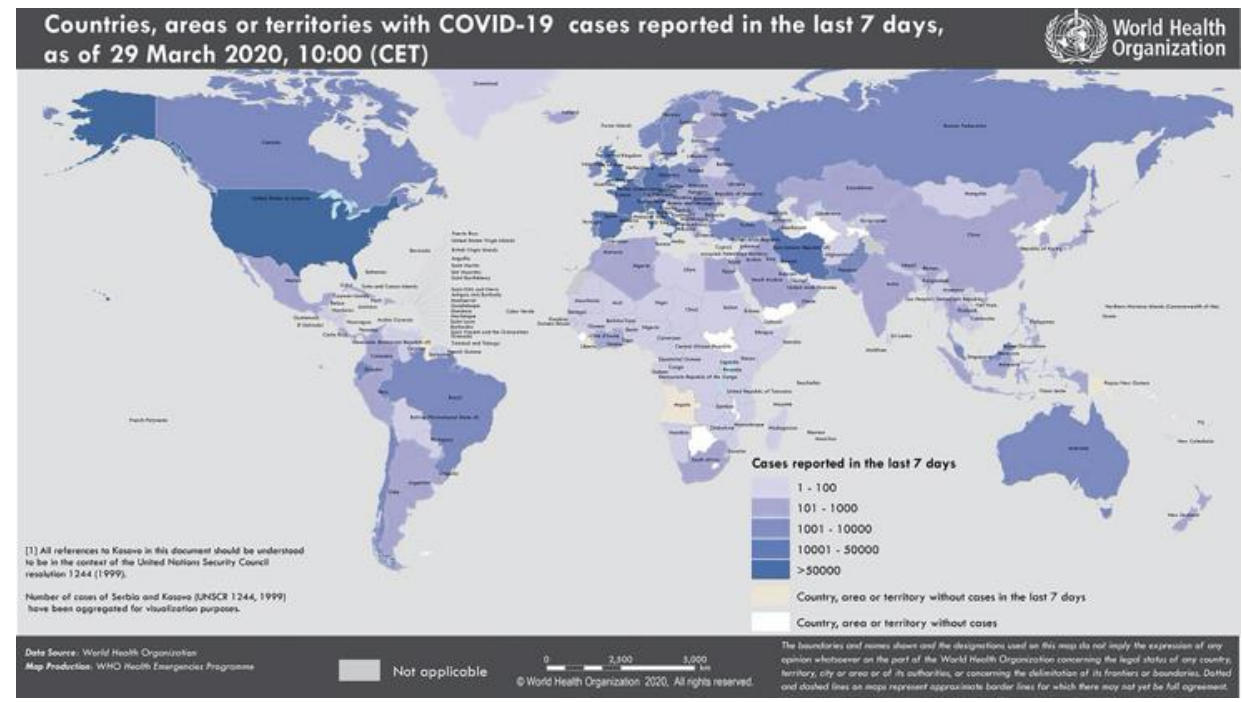

Estatísticas diárias de detecção de novos casos também tem sido divulgadas pela OMS (WHO, 2020b) (Fig. 3). A China tem conseguido reduzir os quadros de novas infecções desde 12 de fevereiro, quando teve seu maior pico. Por outro lado, observa-se que o número de novos diagnósticos de COVID-19 continua em expansão nas diferentes zonas geográficas estabelecidas pela OMS, especialmente nas Américas, Mediterrâneo Oriental e Europa, conforme se observa de 24 de fevereiro a 29 de março. Esses dados reforçam a necessidade de manutenção de medidas de saúde na tentativa de conter a disseminação do vírus.

Figura 3. Curva Epidêmica de casos de COVID-19 confirmados, por dia de notificação e estratificação de regiões da OMS até 29 de março de 2020. (Fonte: WHO, 2020b).

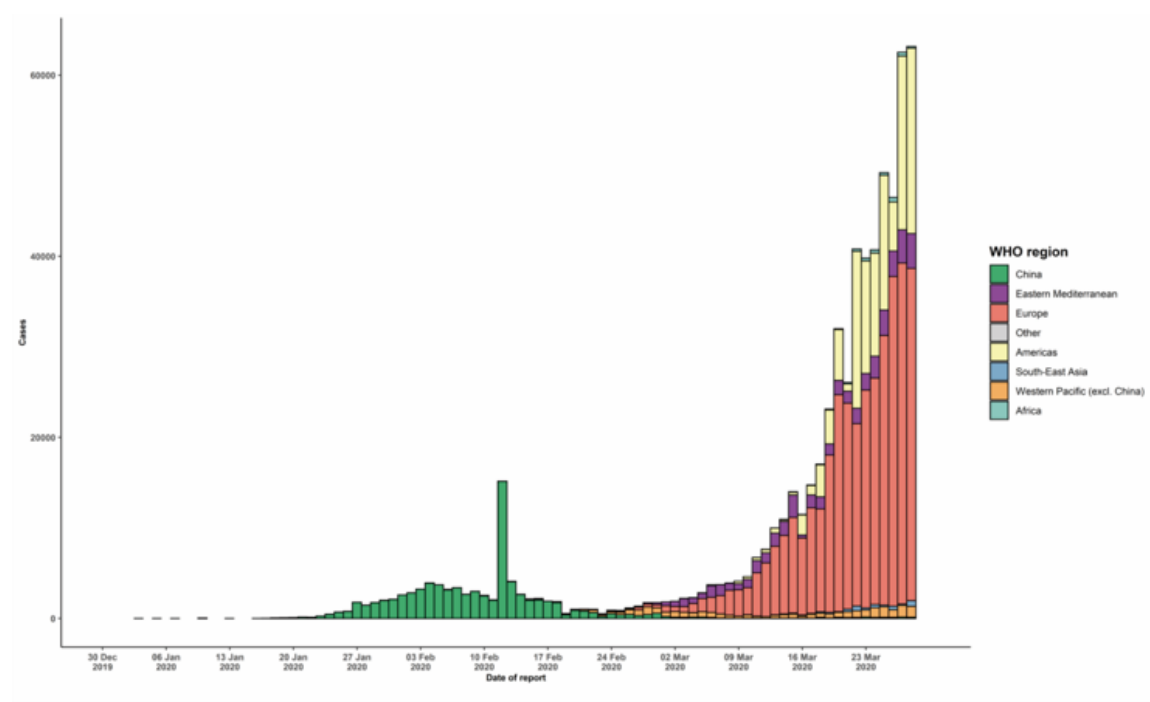

CENÁRIO EPIDEMIOLÓGICO NO BRASIL

No dia 07 de janeiro de 2020, a Secretaria de Vigilância Sanitária (SVS), vinculada ao Ministério da
Saúde, elaborou o primeiro relatório interno para gestores com referência ao novo coronavírus. No dia 08 de janeiro, realizou-se a avaliação de risco utilizando 
os termos do Regulamento Sanitário Internacional (RSI) do impacto no país e no dia 10, inclusão do evento para monitoramento no Comitê de Monitoramento de Eventos. Sendo assim, houve reunião da equipe técnica para a elaboração do protocolo de vigilância com a publicação do primeiro boletim epidemiológico referente ao CODIV-19 no dia 17 de janeiro (MINISTÉRIO DA SAÚDE, 2020a).

Entre 03 e 27 de janeiro de 2020, o Centro de informações Estratégicas de Vigilância em Saúde (CIEVS) recebeu grande quantidade de rumores de pessoas com a possibilidade de contaminação por SARS-CoV-2, entretanto, apenas 127 foram analisados por se tratar de casos com real possibilidade contaminação. Entre os dias 18 e 27 de janeiro, a Secretaria de Vigilância Sanitária (SVS) recebeu notificação de 10 casos para investigação, mas apenas um caso se enquadrava na definição de caso suspeito. Tratava-se de uma estudante de 22 anos que viajou para a cidade de Wuhan. O mesmo foi conduzido para isolamento respiratório em um Hospital na cidade de Belo Horizonte (MINISTÉRIO DA SAÚDE, 2020b).

De 03 de janeiro a 07 de fevereiro de 2020, o Centro de Informações Estratégicas de Vigilância em Saúde (CIEVS) Nacional recebeu 85.229 rumores, entretanto, apenas 238 eram suspeitos de infecção humana pelo novo coronavírus. O principal critério estabelecido naquele momento para apreciação dos casos foi a realização de viagem recente a países com casos confirmados, sendo apenas 18 amostras testadas (MINISTÉRIO DA SAÚDE, 2020b). As seguintes técnicas foram adotadas para o diagnóstico laboratorial de Coronavírus: detecção do genoma viral por meio das técnicas de RT-PCR em tempo real e sequenciamento parcial ou total do genoma viral.

O primeiro caso confirmado de COVID-19 no Brasil foi de um brasileiro de 61 anos, que esteve em
Lombardia, região norte da Itália. O mesmo chegou ao Brasil em 21 de fevereiro de 2020, na cidade de São Paulo/SP. Este também foi o primeiro caso notificado na América Latina (RODRIGUEZ-MORALES et al., 2020).

Após um mês da confirmação do primeiro caso de coronavírus no Brasil, todos os estados registraram casos da doença. Em relação à mortalidade, foram registrados casos nos estados do AM, GO, PE, RJ, RS, SC e SP. Além disso, houve a liberação de 1 bilhão de reais aos estados e municípios para o fortalecimento das ações destinadas ao combate ao vírus (AQUINO, 2020).

Até 28 de março de 2020, foram confirmados 3.904 casos no Brasil, com 114 mortes, perfazendo um total de $2,4 \%$ de letalidade. A região Sudeste concentrava $57 \%$ dos casos (2222), seguida pela região Nordeste com 16\% (624), Sul com 13\% (514), 9\% (184) na região Centro-oeste e 5\% na região Norte (184) (MINISTÉRIO DA SAÚDE, 2020c).

A partir dos dados de modelagem estatística do Imperial College London, os cenários para a COVID19 no Brasil foram alarmantes (Tab. 1). A previsão, publicada em 26 de abril de 2020, levou em consideração os dados de contágio obtidos em países afetados, estatística de hospitalização, estudos referentes à disseminação do vírus em diferentes ambientes, mortalidade e capacidade hospitalar. Os dados foram estimados em diferentes cenários: sem mitigação (sem qualquer tipo de intervenção), com mitigação total (isolamento social de uma parte da população), mitigação reforçada (isolamento social da maior parte da população e cuidado maior com a população idosa) e supressão (testagem e isolamento dos casos positivos, bem como distanciamento social de toda população) (WALKER et. al, 2020). 
Comparando os cenários sem mitigação (sem qualquer intervenção) e supressão precoce (o cenário "ideal”), a diferença no número de mortes chegaria a 1,1 milhão. Além disso, o percentual médio de pacientes hospitalizados em estado grave para os diferentes cenários seria de $26,4 \%$. Como observado, estratégias mais rígidas de controle da infeção e testagem em massa de infectados pode diminuir significativamente a disseminação da doença e reduzir o colapso do sistema de saúde brasileiro.

Tabela 1. Previsão de pessoas infectadas e mortes no Brasil de acordo com o grau de intervenção.

\begin{tabular}{ccccc}
\hline Cenário & Infectados & Mortes & Hospitalizados & Graves (UTI) \\
\hline Sem mitigação & 187.799 .806 & 1.152 .283 & 6.206 .514 & 1.527 .536 \\
Mitigação total & 122.025 .818 & 627.047 & 3.496 .359 & 831.381 \\
Mitigação reforçada & 120.836 .850 & 529.779 & 3.222 .096 & 702.497 \\
Supressão Tardia & 49.599 .016 & 206.087 & 1.182 .457 & 460.361 \\
Supressão precoce & 11.457 .197 & 44.212 & 250.182 & 57.423 \\
\hline
\end{tabular}

Os dados atuais referentes aos números de casos no Brasil podem estar subnotificados, seja pela presença de pessoas assintomáticas ou pela vulnerável política de testes confirmatórios disponibilizada no país, já que não há indicação ou disponibilidade de testes diagnósticos para testagem massiva para a COVID-19. As recomendações atuais do Ministério da Saúde para testagem incluem apenas pessoas com sintomas graves da doença. Segundo Jucá (2020), a quantidade disponível de testes atualmente é insuficiente para a população brasileira. Há relatos de pacientes que receberam o diagnóstico após sete dias. No estado do Rio de janeiro, por exemplo, a cada 100 pacientes, apenas 14 são identificados. Em contramão, países que realizaram a testagem em massa da população, tiveram grande sucesso no controle da pandemia.

\section{ESTRATÉGIAS PARA CONTENÇÃO DA EPIDEMIA POR COVID-19}

Medidas extensivas para reduzir a transmissão de COVID-19 de pessoa para pessoa foram implementadas para controlar o surto atual, tais como o uso de máscaras, práticas de higiene das mãos, prevenção de contatos públicos, detecção de casos, rastreamento de contatos e quarentenas. Até o momento, nenhum tratamento antiviral específico se mostrou eficaz; portanto, as pessoas infectadas dependem principalmente de tratamento sintomático e cuidados de suporte. Atenção e esforços especiais para proteger ou reduzir a transmissão devem ser aplicados em populações suscetíveis, incluindo crianças, profissionais de saúde e idosos (ADHIKARI et al., 2020). Abaixo seguem os objetivos estratégicos de resposta à epidemia por COVID-19 elencados pela OMS (WHO, 2020b):

- Interromper a transmissão de humano para humano, incluindo a redução de infecções secundárias entre contatos próximos e profissionais de saúde, prevenindo eventos de amplificação da transmissão e impedindo a disseminação internacional através de ações coordenadas que garantam a rápida identificação, diagnóstico e manejo dos casos, identificação e acompanhamento dos contatos, prevenção e controle de infecções nos serviços de saúde, implementação de medidas de saúde para 
viajantes, maior conscientização da população e comunicação de risco.

- Identificar, isolar e cuidar dos pacientes precocemente, inclusive fornecendo atendimento otimizado aos pacientes infectados;

- Identificar e reduzir a transmissão da fonte animal;

- Abordar incógnitas cruciais em relação à gravidade clínica, extensão da transmissão e infecção, opções de tratamento e acelerar o desenvolvimento de diagnósticos, tratamentos e vacinas;

- Comunicar informações críticas sobre riscos e eventos a todas as comunidades e combater a desinformação;

- Minimizar o impacto social e econômico por meio de parcerias multi-setoriais.

\section{CONCLUSÃO}

Conforme observado, o número de novos casos e sua possível evolução para manifestações graves ainda justificam medidas preventivas de contágio, especialmente ante ao fato de não haver tratamento ou vacina com comprovada eficácia clínica e da baixa capacidade do Brasil, em especial, em identificar casos positivos assintomáticos, os quais podem contribuir para a disseminação do vírus. Desta forma, as recomendações com embasamento científico promovidas pelas agências de saúde precisam ser obedecidas no sentido de minimizar o impacto da doença em todo o mundo, principalmente no Brasil. Adicionalmente, mais pesquisas de alta qualidade precisarão fornecer formas válidas e confiáveis de gerenciar esse tipo de emergência de saúde pública, tanto a curto quanto a longo prazo.

\section{AGRADECIMENTO}

Agradecemos em especial aos profissionais de saúde envolvidos no combate à esta epidemia e a toda comunidade científica mundial que não tem envidado DOI: http://dx.doi.org/10.20873/uftsuple2020-8710 2020 esforços em divulgar e pesquisar novos caminhos para a contenção e tratamento dos pacientes infectados.

Todos os autores declararam não haver qualquer potencial conflito de interesses referente a este artigo.

\section{REFERÊNCIAS}

ADHIKARI, S.P.; MENG, S.; WU, Y.; MAO, Y.; YE, R.; WANG, Q.; SUN, C.; SYLVIA, S.; ROZELLE, S.; RAAT, H.; ZHOU, H. Epidemiology, causes, clinical manifestation and diagnosis, prevention and control of coronavirus disease (COVID-19) during the early outbreak period: a scoping review. Infectious Diseases of Poverty, v. 9, n. 1, p. 29, 2020.

AQUINO, V. 2020. Ministério de Saúde. Brasil registra 2.915 casos confirmados de coronavírus e 77 mortes. Disponível em: https://www.saude.gov.br/noticias/agenciasaude/46610-brasil-registra-2-915-casosconfirmados-de-coronavirus-e-77-mortes. Acesso em: $26 / 03 / 2020$

BAI, Y.; YAO, L.; WEI, T.; TIAN, F.; JIN, D.; CHEN, L.;WANG, M. Presumed asymptomatic carrier transmission of COVID-19. JAMA, 2020. DOI:10.1001/jama.2020.2565.

CHAN, J.; YUAN, S.; KOK, K.; TO, K.K.; CHU, H.; YANG, J.; XING, F.; LIU, J.; YIP, C.C.; POON, R.W.; TSOI, H.; LO, S.K.; CHAN, K.; POON, V.K.; CHAN, W.; IP, J.D.; CAI, J.; CHENG, V.C.; CHEN, H.; HUI, C.K.; YUEN, K. A familial cluster of pneumonia associated with the 2019 novel coronavirus indicating person-to-person transmission: a study of a family cluster. The Lancet, v. 395, n. 10223, p. 514523, 2020.

GUAN, W.; NI, Z.; HU, Y.; LIANG, W.; OU, C.; HE, J.; LIU, L.; SHAN, H.; LEI, C.; HUI, D.; DU, B.; LI, L.; ZENG, G.; YUEN, K.; CHEN, R.; TANG, C.; WANG, T.; CHEN, P.; XIANG, J.; LI, S.; WANG, J.; LIANG, Z.; PENG, Y.; WEI, L.; LIU, Y.; HU, Y.; PENG, P.; WANG, J.; LIU, J.; CHEN, Z.; LI, G.; ZHENG, Z.; QIU, S.; LUO, J.; YE, C.; ZHU, S.; ZHONG, N. Clinical characteristics of 2019 novel coronavirus infection in China. New England Journal of Medicine, 2020. DOI: 10.1056/NEJMoa2002032

JHUM (Johns Hopkins University \& Medicine). 2020. Coronavirus resource center. Disponível em: https://coronavirus.jhu.edu/map.html. Acesso em: 29 de março de 2020.

Revista Desafios - v7, n. Supl. COVID-19, 
JUCÁ, B. 2020. Com gargalo de testes para coronavírus, Brasil vê só a ponta do iceberg com seus 2.201 casos e 46 mortes. Disponível em: https://brasil.elpais.com/brasil/2020-03-24/comgargalo-de-testes-para-coronavirus-brasil-ve-so-aponta-do-iceberg-com-seus-2201-casos-

de46mortes.html. Acesso em: 29/03/2020.

MINISTÉRIO DA SAÚDE. 2020a. Boletim Epidemiológico: Novo coronavírus (2019-nCoV). Disponível em: https://portalarquivos2.saude.gov.br/images/pdf/2020 /janeiro/23/Boletim_epidemiol ogico_SVS_04.pdf. Acesso em 27/03/2020.

MINISTÉRIO DA SAÚDE. 2020b. Boletim Epidemiológico: Infecção Humana pelo Novo Coronavírus (2019-nCoV). Disponível em: https://portalarquivos2.saude.gov.br/images/p df/2020/janeiro/28/ Boletim-epidemiologico-SVS28jan20.pdf. Aceso em 27/03/2020.

MINISTÉRIO DA SAÚDE. 2020c. Painel Coronavírus. Disponível em: https://covid.saude.gov.br/. Acesso em: 28/03/2020

RODRIGUEZ-MORALES, A.; BONILLAALDANA, D.; TIWARI, R.; SAH, R.; RABAAN, A.; DHAMA, K. COVID-19, an Emerging Coronavirus Infection: Current Scenario and Recent Developments - An Overview. Journal of Pure and Applied Microbiology. v.14, n. 6150, p. 05-12, 2020.

ROTHAN, H.A.; BYRAREDDY, S.N. The epidemiology and pathogenesis of coronavirus disease (COVID-19) outbreak. Journal of Autoimmunity, p. 102433, 2020. DOI: 10.1016/j.jaut.2020.102433.

VELAVAN, T.P.; MEYER, C.G. The COVID-19 epidemic. Tropical Medicine \& International Health, v. 25, n. 3, p. 278-280, 2020.

WALKER, P.; WHITTAKER, C.; WATSON, O.; BAGUELIN, M.; AINSLIE, K.; BHATIA, S.; BHATT, S.; BOONYASIRI, A.; BOYD, O.; CATTARINO, L.; CUCUNUBÁ, Z.; CUOMODANNENBURG, G,; DIGHE, A.; DONNELLY, C,; DORIGATTI, I.; ELSLAND, S.; FITZJOHN, R.;
FLAXMAN, S.; FU, H.; GAYTHORPE, K.; GEIDELBERG, L.; GRASSLY, N.; GREEN, W.; HAMLET, A.; HAUCK, K.; HAW, D.; HAYES, S.; HINSLEY, W.; IMAI, N.; JORGENSEN, D.; KNOCK, E.; LAYDON, D.; MISHRA, S.; NEDJATIGILANI, G.; OKELL, L.; RILEY, S.; THOMPSON, H.; UNWIN, J.; VERITY, R.; VOLLMER, M.; WALTERS, C.; WANG, H.; WANG, Y.; WINSKILL, R.; XI, X.; FERGUSON, N.; GHAN, Z. 2020. The Global Impact of COVID-19 and Strategies for Mitigation and Suppression. Disponível em: https://www.imperial.ac.uk/media/imperialcollege/medicine/sph/ide/gida-fellowships/ImperialCollege-COVID19-Global-Impact-26-03-2020v2.pdf. Acesso em: 27/03/2020.

WHO (WORLD HEALTH ORGANIZATION). 2020a. Coronavirus disease 2019 (COVID-19) situation reports. Disponível em: https://www.who.int/emergencies/diseases/novelcoronavirus-2019/situation-reports. Acesso em: 29 de março de 2020.

WHO (WORLD HEALTH ORGANIZATION). 2020b. Coronavirus disease 2019 (COVID-19) situation reports-69. Disponível em: https://www.who.int/docs/defaultsource/coronaviruse/situation-reports/20200329sitrep-69-covid-19.pdf?sfvrsn=8d6620fa_8. Acesso em: 29 de março de 2020.

WU, F.; ZHAO, S.; YU, B.; CHEN, Y.; WANG, W.; SONG, Z.; HU, Y.; TAO, Z.; TIAN, J.; PEI, Y.; YUAN, M.; ZHANG, Y.; DAI, F.; LIU, Y.; WANG, Q.; ZHENG, J.; XU, L.; HOLMES, E.C.; ZHANG, Y. A new coronavirus associated with human respiratory disease in China. Nature, v. 579, n. 7798, p. 265-269, 2020.

ZHOU, P.; YANG, X.; Wang, X; HU, B.; ZHANG, L.; ZHANG, W.; SI, H.; ZHU, Y.; LI, B.; HUANG, C.; CHEN, H.; CHEN, J.; LUO, Y.; GUO, H.; JIANG, R.; LIU, M.; CHEN, Y.; SHEN, X.; WANG, X. ZHENG, X.; ZHAO, K.; CHEN, Q.; DENG, F.; LIU, L.; YAN, B.; ZHAN, F.; WANG, Y.; XIAO, G.; SHI, Z. A pneumonia outbreak associated with a new coronavirus of probable bat origin. Nature, v. 579, n. 7798 , p. $270-273,2020$ 